\title{
THERMALLY ISOLATED MICROMACHINED QUARTZ RESONATOR ARRAY FOR DIFFERENTIAL MICRO-CALORIMETRIC MEASUREMENTS

\author{
David Gaddes $^{l}$ and Srinivas Tadigadapa ${ }^{1,2}$
} \\ ${ }^{1}$ Department of Biomedical Engineering, The Pennsylvania State University, USA \\ ${ }^{2}$ Department of Electrical Engineering, The Pennsylvania State University, USA
}

\section{INTRODUCTION}

We have reported on the concept and performance of a calorimetric biosensor using a micromachined Y-cut quartz resonator as sensitive thermometer and a physically decoupled but closely located microfluidic reaction chamber[1]. Using layer by layer urease enzyme immobilization and combining with microfluidic pumping of the analyte we were able to demonstrate continuous measurements of urea with resolution of $\sim 2 \mathrm{mM}$ [2]. Micromachined bulk acoustic wave resonators with frequencies in few hundred $\mathrm{MHz}$ allow for temperature resolution of tens of microKelvin and better SNR as compared to thin film thermistors and thermocouples [1]. Proximity coupling of the thermal energy via conduction through thin film of air and radiation from the reaction chamber to the resonator thermometer, allows for complete separation of the biology and the physical sensor/electronics. In this paper we report on over 18 times improvement of this calorimetric sensor design achieved through improved thermal isolation of the resonators and differential sensor configuration to remove common mode temperature artifacts.

\section{RESULTS AND DISCUSSION}

8-resonator arrays of monolithic, micromachined quartz resonators of thickness $10 \mu \mathrm{m}(167 \mathrm{MHz})$ were fabricated using RIE etching of quartz [3] as schematically shown in Fig. 1. The inverted mesa design of the resonators resulted in large thermal coupling and cross-talk between the resonators. Focused ion beam etching was used to create a thermally isolated resonator pixel (Fig. 1). The thermally isolated resonator was used as the reference sensor which provides a sensitive reference for background temperature changes as well as cancels out any spurious noise. For these measurements a $1 \mathrm{~mm}$ diameter Kapton $^{\circledR}$ tubing with wall thickness of $25 \mu \mathrm{m}$ was used. A nichrome wire of $4 \Omega$ resistance was wound and placed upstream in the tube and the temperature of the water downstream from the heater was measured by inserting a thermocouple into the tube. Two such Kapton ${ }^{\circledR}$ tubes (channels) are placed above the resonator chip (Fig. 2). Although only channel 1 was exposed to heated water $(6.05 \mathrm{~mW}$ for $20 \mathrm{~s})$, in the case where the resonator is not thermally isolated, heat is conducted through the quartz substrate and results in the temperature of both resonators to rise and results in poor compensation in the differential mode (Fig. 3(a)). For the thermally isolated resonator chip, when only channel 1 was exposed to heat $(450 \mu \mathrm{W}, 20 \mathrm{~s})$, excellent compensation in the differential mode is achieved (Fig. 3(b)). Similarly, when a common mode thermal signal $(3.0 \mathrm{~mW}, 20 \mathrm{~s})$ is applied simultaneously to both channels, excellent compensation is achieved in the thermally isolated sensor chip (Fig. 3(c)). In this case the reference sensor effectively cancels both thermal drift as well as the large $\sim 175 \mathrm{~Hz}$ common mode response. Allan deviation noise characteristics of similar frequency Y-cut and AT-cut resonators are compared in Fig. 4. The noise in Y-cut resonator is 10 times larger mainly due to radiatively and conductively induced thermal response. Fig. 5 shows the thermal sensitivity of various Y-cut resonators, showing 2.25 times higher sensitivity for the $165 \mathrm{MHz}$ resonator in comparison to a $68 \mathrm{MHz}$ resonator. Furthermore, the $167 \mathrm{MHz}$ thinner and thermally isolated resonator with IR reflective gold electrode has 1.76 times lower noise than $68 \mathrm{MHz}$ Y-cut resonator thereby improving the overall SNR by 4.73 times. Combining the improved differential measurement achieved by the thermally isolated sensor with the improved SNR of the $167 \mathrm{MHz}$ resonator, an improved resolution of $110 \mu \mathrm{W}$ from the previous limit of 2 $\mathrm{mW}$ measured for a non-isolated $90 \mathrm{MHz}$ resonator is realized. The improved resonator design is expected to enable quantification of urea down to $275 \mu \mathrm{M}$ where previously only 5 $\mathrm{mM}$ was possible.

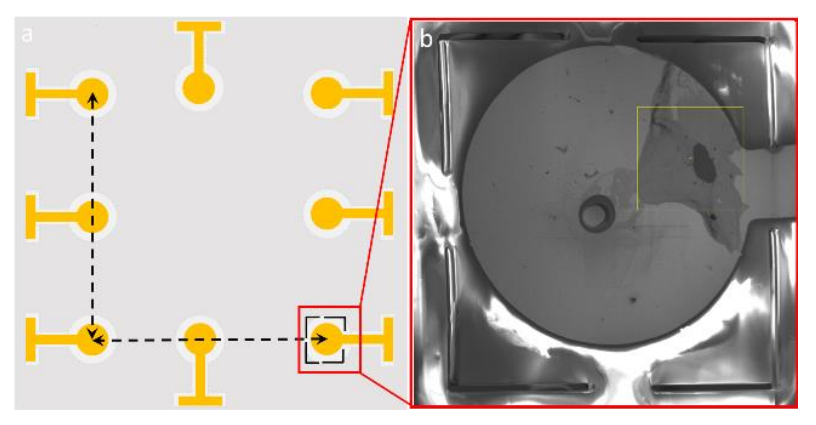

Figure 1: (a) Schematic 8-pixel Y-cut quartz resonator array (b) Fabricated device with thermal isolation cuts along the marked lines

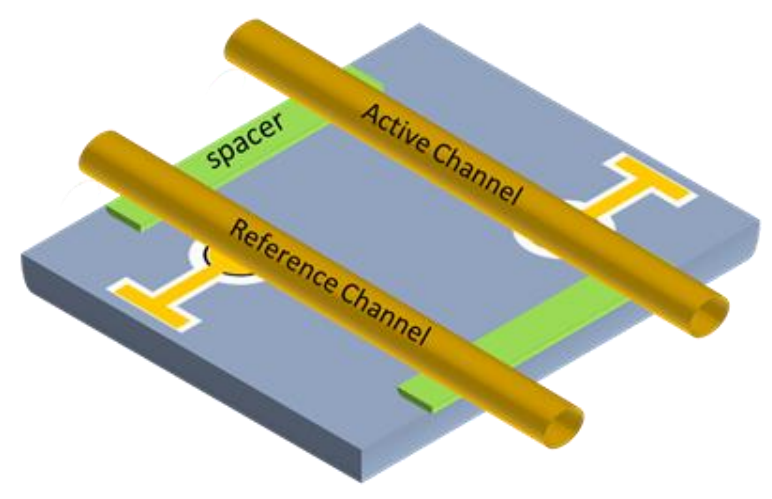

Figure 2: Schematic of the dual channel calorimetric sensing system.
Solid-State Sensors, Actuators and Microsystems Workshop Hilton Head Island, South Carolina, June 5-9, 2016 

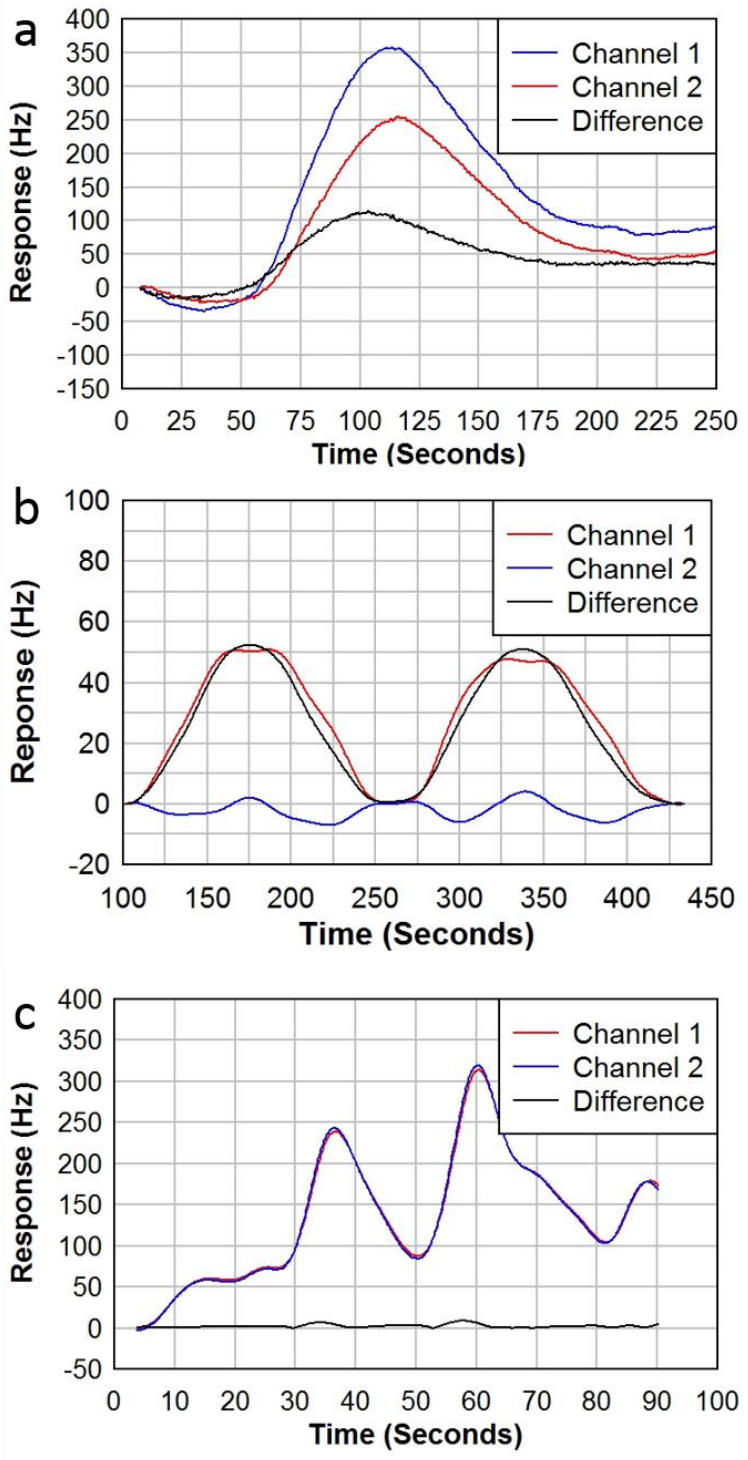

Figure 3: Differential measurement based compensation: (a) Resonator array (without thermal isolation). Heated solution in channel 1 and channel 2 acts as a reference. (b) Resonator array with thermally isolated resonator under channel 2 acts as a reference. Heated solution in channel 1 (c) Common mode thermal signal compensation using the thermally isolated resonator array.

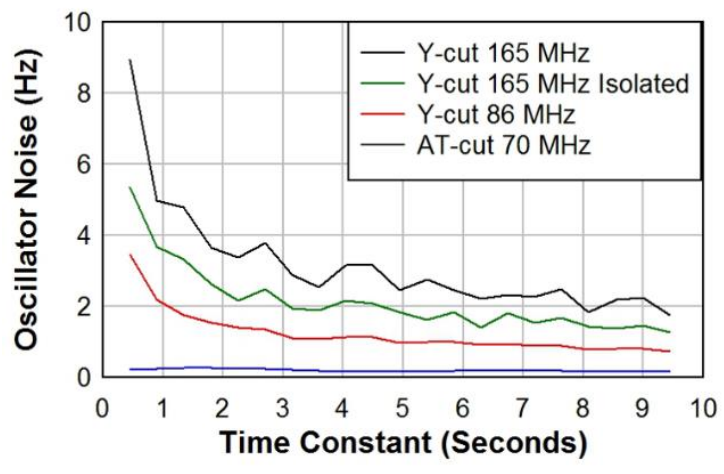

Figure 4: Allan Deviation of Y-cut resonators at $68 \mathrm{MHz}$ (red), a $70 \mathrm{MHz}$ AT cut quartz resonator (blue), a $165 \mathrm{MHz}$ resonator (black) and an isolated $165 \mathrm{MHz}$ resonator (green).

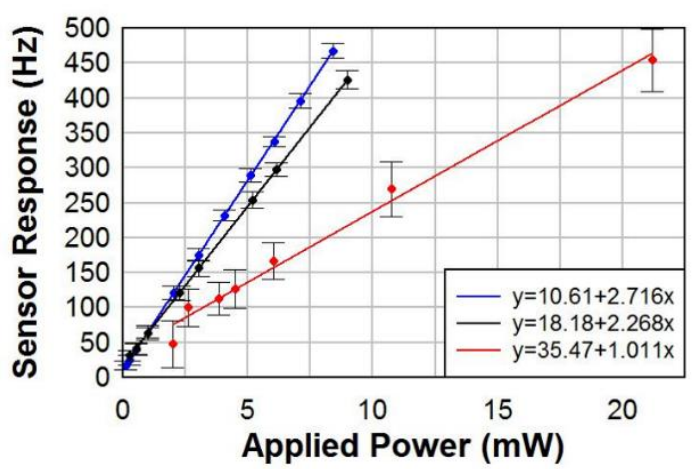

Figure 5: The sensitivity of a non-isolated $68 \mathrm{MHz}$ resonator (red) and $167 \mathrm{MHz}$ resonator (black) are compared with that of a thermally isolated $165 \mathrm{MHz}$ resonator (blue).

\section{References:}

[1] K. Ren, P. Kao, M. B. Pisani, and S. Tadigadapa, "Monitoring biochemical reactions using Y-cut quartz thermal sensors," Analyst, vol. 136, no. 14, pp. 2904 2911, 2011.

[2] D. E. Gaddes, M. C. Demirel, W. B. Reeves, and S. Tadigadapa, "Remote calorimetric detection of urea via flow injection analysis.," Analyst, vol. 140, no. 23, pp. 8033-40, 2015.

[3] Hatipoglu, Gokhan, and Srinivas Tadigadapa. "Experimental studies in magnetically induced transverse force-frequency effect in thin quartz microresonators." Journal of Applied Physics 118.3 (2015): 034508. 